\title{
Brightness enhancement in Non-Hermitian VCSELs
}

\author{
Waqas W. Ahmed ${ }^{1}$, Ramon Herrero ${ }^{2}$, Muriel Botey ${ }^{2}$, Ying Wu $\mathbf{u}^{1}$, Kestutis Staliunas ${ }^{2,3}$ \\ 1. Division of Computer, Electrical and Mathematical Sciences and Engineering, King Abdullah University of Science and Technology \\ (KAUST), Thuwal, 23955-6900, Saudi Arabia \\ 2. Departament de Física, Universitat Politècnica de Catalunya (UPC), Colom 11, E-08222 Terrassa, Barcelona, Spain \\ 3. Institució Catalana de Recerca i Estudis Avançats (ICREA), Passeig Lluís Companys 23, E-08010, Barcelona, Spain
}

Vertical Cavity Surface Emitting lasers (VCSELs) are compact and efficient light sources useful for a variety of applications. However, due to lack of a transverse mode control mechanism, such lasers suffer from poor spatial beam quality, intrinsic spatiotemporal instabilities and nonlinear destabilizing effects such as filamentation and spatial hole burning [1]. Therefore, there is a need for new strategies to manipulate the light wave dynamics to enhance the stability of VCSELs. Recently, non-Hermitian media have become a flexible platform for new functionalities such as asymmetric coupling, unidirectional invisibility, single mode lasing [2-3]. In this presentation, we propose a novel stabilization mechanism for VCSLEs to obtain bright and narrow beams. The mechanism relies on non-Hermitian configuration of the laser potential, achieved by simultaneous spatial modulation of the refractive index and gain-loss profiles. In particular, we consider axisymmetric non-Hermitian potentials expressed as: $n(r)=n_{R} \cos (q r)-i n_{I} \cos (q r-\phi)$ where $n_{R}$ and $n_{I}$ are the amplitude of the refractive index and gain-loss modulations, and $\phi$ is the relative phase shift between them. Such potentials may confine the emitted light around the central part of VCSELs, through unidirectional-inward radial coupling among the transverse modes [4]. The interplay of the relative strength and relative phase of the index and gain-loss modulations manipulate the wave dynamics of such lasers to emit powerful and narrow beams of high brightness. We use the mean-field paraxial model to study the spatiotemporal dynamics of such VCSELs with non-Hermitian potentials. The output emission of conventional VCSEL and modified VCSEL with concentric non-Hermitian configuration is shown in Fig. 1(a,b), illustrating irregular and stable localized pattern, respectively. We assess the performance through the central intensity enhancement [see Fig. 1(c)] and field concertation [see Fig. 1(d)]. The spatial dynamics for a representative point is provided in Fig. 1(e). The stationary intensity profile and its corresponding cross-section show that intensity is strongly concentrated at $r=0$ due to the favoured radial coupling of inward propagating waves, as depicted in the transverse field flow on the inset [see Fig. 1(e)].

(a)

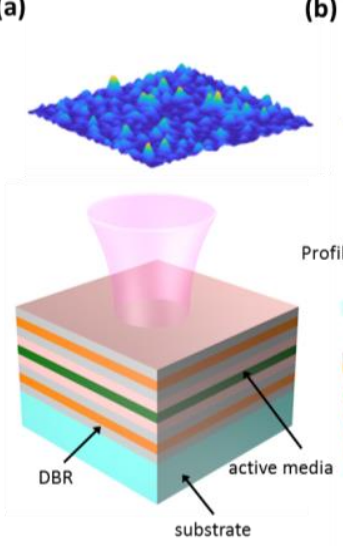

(b)

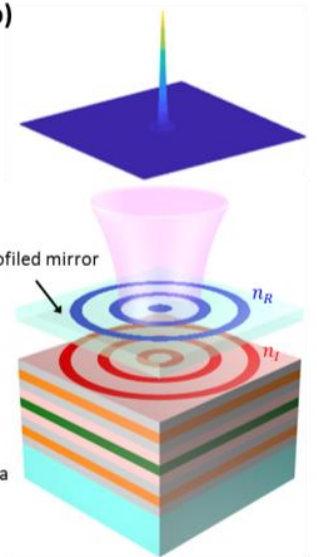

(c)

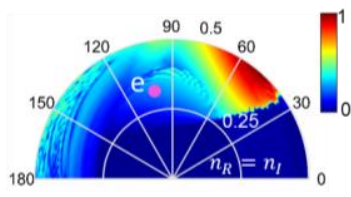

(d)

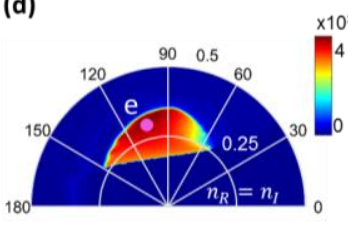

(e)
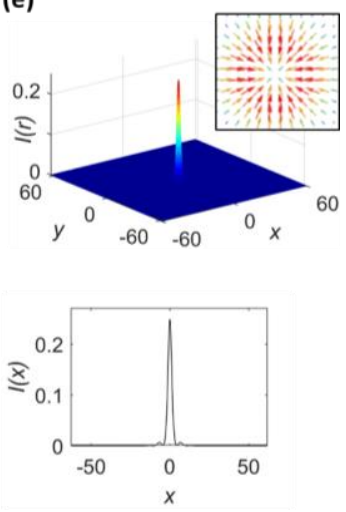

Fig. 1 (a) Complex irregular emission in conventional broad-area VCSEL (b) bight and narrow beam emission in modified VCSEL with non-Hermitian concentric configuration $\left(n_{R}\right.$ : refractive index, $n_{I}$ : gain-loss) (c) Central intensity enhancement (d) axial concentration factor in parameter space $\left(n_{I}, \phi\right)$ for $n_{R}=0.25$ (e) Intensity profile and corresponding axial cross-section in stationary state. The inset illustrates the transverse field flow around the center, showing the unidirectional-inward radial coupling.

We expect the proposed mechanism is applicable to regularize the radiations of other broad aperture and microlasers, which typically emit quite random and irregular light patterns. Besides, the reported concentration effect is universal, and could be extended to random and quasiperiodic background potentials.

\section{References}

[1] J. Othsubo, Semiconductor Lasers: Stability, Instability and Chaos (Springer, Heidelberg, 2013).

[2] C. E. Ruter, K. G. Makris, R. El-Ganainy, D. N. Christodoulides, M. Segev, and D. Kip, "Observation of parity-time symmetry in optics," Nat. Phys. 6, 192 (2010).

[3] Z. Lin, H. Ramezani, T. Eichelkraut, T. Kottos, H. Cao, and D. N. Christodoulides, "Unidirectional Invisibility Induced by PT-Symmetric Periodic Structures," Phys. Rev. Lett. 106, 213901 (2011).

[4] W. W. Ahmed, R. Herrero, M. Botey, and K. Staliunas, "Locally Parity-Time Symmetric and globally Parity-Symmetric systems," Phys. Rev. A 94, 053819 (2016). 